\title{
Une instance parallèle
}

La dignité humaine, l'autonomie, la justice, l'interdiction de faire du mal et le devoir de faire du bien sont les principes centraux mentionnés dans le commentaire explicatif accompagnant le projet d'un nouvel article constitutionnel 118a consacré à la recherche sur l'être humain. Les rapports issus de la procédure de consultation comportent 167 pages et ne sont que le premier maillon d'un débat à longue échéance qui ne s'achèvera qu'en 2010 au moment de l'entrée en vigueur de la loi. Le but visé consiste à trouver une solution fédérale uniforme et définitive pour réglementer la recherche dans les domaines suivants: médecine, psychologie clinique, biologie humaine et sciences sociales. Est considérée comme recherche visant l'acquisition de connaissances selon des critères scientifiques, la recherche sur des personnes (essais cliniques), sur du matériel humain (organes, tissus, cellules), sur des données personnelles (médicales, génétiques), sur des cadavres ou sur des embryons et des fœtus humains. Les exigences en matière de projets de recherche effectués sur des personnes incapables de discernement (enfants, patients atteints de démence) ou dans des situations d'urgence sont plus strictes, de même que pour les projets impliquant des femmes enceintes ou des archives de matériel corporel ou de biobanques (p.ex. «Sesam»: Swiss Etiological Study of Adjustment and Mental Health). Une interdiction globale proscrit en outre le paiement des dons d'organes ou la commercialisation des organes humains.

\section{Matière à légiférer ...}

Depuis la publication du code de Nuremberg en 1947, un ensemble de règles juridiques réglemente la recherche biomédicale. La déclaration d'Helsinki de 1964 de l'Association médicale mondiale a été déterminante pour l'élaboration de normes visant à protéger les personnes faisant l'objet de recherches. Cette déclaration a été rendue obligatoire par la Convention sur les droits de l'homme et la biomédecine du Conseil de l'Europe de 1997, ainsi que par son protocole additionnel de 2005. La Suisse a signé ladite convention mais ne l'a pas encore ratifiée. La Confédération est certes responsable de certains domaines partiels tels que les médicaments, la santé, la procréation médicalement assistée, le génie génétique, la médecine de transplantation et, par le biais du droit civil et pénal, de toute question concernant l'information et l'approbation du patient. Il n'existe cependant aucune norme spécifique qui règle les compétences en matière de recherche sur l'être humain, même si cette dernière touche à plusieurs droits fondamentaux. L'article $7 \mathrm{de}$ la Constitution fédérale pose la dignité humaine en tant que principe constitutionnel suprême de l'ordre juridique fédéral. Il s'agit du principe à la base de tous les droits fondamentaux, dont font notamment aussi partie le droit à la vie, la liberté individuelle et la pro- tection de la sphère privée. Etant donné que la liberté de l'enseignement et de la recherche scientifiques est également un droit constitutionnel, toute limitation apportée dans ce domaine nécessite une pesée d'intérêts.

\section{... et questions d'éthique}

L'éthique porte sur des actions et des prises de décision qui soient en accord avec la morale. Les juristes prescrivent ce que nous avons le droit de faire, les spécialistes en éthique décrivent les valeurs morales courantes ou établissent des normes pour régir notre comportement. Ces derniers ont le vent en poupe, car on consulte des «éthiciens» pour toute question dont la réponse comporte un dilemme. Chaque entreprise, chaque secteur professionnel, la Confédération et les cantons, et bientôt chaque clinique ayant une certaine importance, financent, que ce soit prescrit par la loi ou qu'il s'agisse d'une décision volontaire, les gardiens de la morale des conseils d'éthique. La loi relative à la recherche sur l'être humain actuellement en discussion envisage deux modèles. Le premier prévoit que des commissions cantonales d'éthique se chargent de faire appliquer la loi comme à l'accoutumée et la seconde, la création de commissions régionales compétentes dont les membres sont élus par la Confédération. Ces deux options comportent des chances et des risques. Une fondation de droit privé comme l'Académie suisse des sciences médicales a, par de nombreuses directives, prouvé son utilité. L'on peut toutefois se demander à quoi sert la pléthore croissante de conseillers et de rhétoriciens passés maîtres dans les arguties. Dans le meilleur des cas, ils ordonnent les faits et collectent des arguments pour les débats publics et, dans la pire des hypothèses, ils font office d'instance parallèle dont les décisions manquent de transparence et engendrent véritablement des difficultés. L'équilibre soigneusement pondéré entre Etat et pouvoir, fruit conjoint de la politique, des lois, des tribunaux et du droit démocratique, ne laissait jusqu'à présent pas de place à d'autres acteurs ayant des pouvoirs si étendus. Le risque existe que des problèmes gênants et impopulaires ne soient pas résolus par les organes responsables, mais qu'on les fasse endosser à ces «commission de veilleurs de nuit». Pour le débat public, nous pouvons compter sur les médias et les partis politiques et pour la pondération des intérêts, sur les juristes. Une commission d'éthique représente certes une voix parmi d'autres, dont on peut espérer qu'elle sera sage et utile, mais il s'avère que la dynamique inhérente à toute instance vise à lui permettre d'étendre et de renforcer sa position. J'en veux pour preuve le souhait déjà formulé par ces commissions de définir elles-mêmes les critères d'aptitude et de certification à appliquer. Or, pour les questions d'éthique, c'est nous tous qui sommes responsables.

Erhard Taverna 
000 